\title{
Estimativa do estoque de carbono por métodos indiretos em área de restauração florestal em Minas Gerais
}

\author{
Carbon stock estimate using indirect methods \\ in a forest restoration area in Minas Gerais State
}

\author{
Helane França Silva ${ }^{1}$, Sabina Cerruto Ribeiro ${ }^{2}$, Soraya Alvarenga Botelho ${ }^{3}$, \\ Regiane Aparecida Vilas Boas Fariaa ${ }^{3}$, Marcília Bruna Reis Teixeira ${ }^{4}$ e José Marcio Mello ${ }^{3}$
}

\begin{abstract}
Resumo
O presente trabalho foi realizado com o objetivo de estimar e comparar o estoque de carbono obtido por três métodos indiretos em uma área em restauração com 21 anos de idade no sul de Minas Gerais. Dados de inventários florestais (diâmetro e altura) de parcelas permanentes medidas em 2010 e 2013 foram utilizados para estimar a biomassa a partir do uso de uma equação alométrica. A densidade básica da madeira (DBM) e o teor de carbono foram determinados em laboratório para as espécies de maior importância ecológica da área de estudo. A quantificação do estoque de carbono se deu por três métodos indiretos: multiplicação da biomassa seca (BS) pelo teor de carbono obtido em laboratório (M1); multiplicação da BS pelo fator 0,5 (M2) e uso de uma equação alométrica (M3). As espécies que apresentaram o menor e o maior teor de carbono foram Lafoensia vandelliana (43,70\%) e Anadenanthera peregrina (46,60\%). Já para a DBM as espécies que apresentaram o menor e o maior valor foram Cecropia pachystachya $(0,31$ $\mathrm{g} \mathrm{cm}^{-3}$ ) e Anadenanthera peregrina $\left(0,72 \mathrm{~g} \mathrm{~cm}^{-3}\right)$. Os estoques de carbono obtidos pelos métodos 1,2 e 3 foram $49,73 \mathrm{tha}^{-1}, 55,65 \mathrm{t} \mathrm{ha}^{-1}$ e $54,70 \mathrm{t} \mathrm{ha}^{-1}$, respectivamente. Observou-se que o uso da equação alométrica e de um teor de carbono genérico levou a superestimativa do estoque de carbono na área avaliada. Dessa forma, o uso do teor de carbono determinado em laboratório contribuiu para aumentar a precisão da estimativa de estoque de carbono.
\end{abstract}

Palavras-chave: biomassa florestal, teor de carbono, fixação de carbono, incremento, reflorestamento.

\begin{abstract}
This study aimed to estimate and compare the carbon stock obtained from three indirect methods in a reforested area of 21 years in southern Minas Gerais State, Brazil. Forest inventory data (diameter and height) from permanent plots, measured between 2010 and 2013, were used to estimate the biomass (dry weight) using an allometric equation. Basic wood density (BWD) and carbon content were only determined for species with high ecological importance. Carbon stock was obtained by three indirect methods: multiplying dry biomass (DB) by the carbon content (M1) obtained through laboratory analysis; multiplying the DB by the factor 0.5 (M2); and use of an allometric equation (M3). The species with the lowest and highest carbon content were Lafoensia vandelliana (43.70\%) and Anadenanthera peregrina (46.60\%). As for the BWD, the species that showed the lowest and highest value were Cecropia pachystachya $(0.31 \mathrm{~g}$ $\left.\mathrm{cm}^{-3}\right)$ and Anadenanthera peregrina $\left(0.72 \mathrm{~g} \mathrm{~cm}^{-3}\right)$. Carbon stocks obtained by methods 1,2 , and 3 were $49.73 \mathrm{t} \mathrm{ha}^{-1} ; 55.65 \mathrm{tha}^{-1}$ and $54.70 \mathrm{t} \mathrm{ha}^{-1}$, respectively. The using an allometric equation and generic carbon content overestimated the carbon stock in the studied area. Thus, the use of carbon content determined in laboratory improved the accuracy estimation of the carbon stock.
\end{abstract}

Keywords: forest biomass, carbon content, carbon fixation, increment, reforestation.

\section{INTRODUÇÃO}

A restauração florestal tem um papel importante na mitigação das mudanças climáticas. O plantio de árvores em áreas antes degradadas, além de restabelecer a diversidade biológica e os processos

\footnotetext{
${ }^{1}$ Professora Assistente. UFPI - Universidade Federal do Piauí. Campus Professora Cinobelina Elvas - Rodovia Municipal Bom Jesus - Viana, Km 01 - Planalto Horizonte - 64900-000 - Bom Jesus, PI. E-mail: helane.engflo@gmail.com

2Professora Adjunto. UFAC - Universidade Federal do Acre - Centro de Ciências Biológicas e da Natureza. Distrito Industrial - 69920-900 - Rio Branco, AC. E-mail: sabina.ufac@gmail.com

3Professor(a) Associado(a). UFLA - Universidade Federal de Lavras. Caixa Postal, 37 - 37200-00 - Lages, MG - E-mail: sbotelho@dcf.ufla.br; josemarcio@dcf.ufla.br

${ }^{4}$ Doutoranda em Estatística e Experimentação Agropecuária. UFLA - Universidade Federal de Lavras. Caixa Postal, 37 37200-00 - Lages, MG. E-mail: marciliabruna@yahoo.com.br
}

Sci. For., Piracicaba, v. 43, n. 108, p. 943-953, dez. 2015 DOI: dx.doi.org/10.18671/scifor.v43n108.18 
ecológicos do ecossistema que fora deteriorado, possibilita uma mudança positiva no balanço do carbono local, graças a capacidade das árvores em sequestrar o $\mathrm{CO}_{2}$ atmosférico e estoca-lo na biomassa (SANQUETTA; BALBINOT, 2004).

Entretanto, para o monitoramento ao longo do tempo do incremento do estoque de carbono em áreas em processo de restauração é necessário o uso de métodos de quantificação dos estoques de biomassa e carbono que gerem estimativas confiáveis (ASHTON et al., 2012). A biomassa florestal pode ser obtida por métodos diretos, que implicam no abate e pesagem de todo o material vegetal arbóreo, ou por métodos indiretos que envolvem o uso de equações alométricas ou imagens de satélite.

Já o estoque de carbono é comumente obtido a partir da multiplicação da biomassa pelo teor de carbono (obtido na literatura ou determinado em laboratório) e pela aplicação de uma equação alométrica. Valores genéricos de teor de carbono obtidos na literatura vêm sendo amplamente utilizados em diversos estudos, sendo comum a adoção do fator 0,5 estabelecido pelo Intergovernmental Panel on Climate Change (IPCC, 2003), o qual considera que $50 \%$ da biomassa seca é composta por carbono (SOARES; OLIVEIRA, 2002; HOUGHTON, 2005; MELO; DURIGAN, 2006; RIBEIRO et al., 2009; ALMEIDA et al., 2010; PAIVA et al., 2011; SOUZA; FIORENTIN, 2013). Mais recentemente, o IPCC estabeleceu o fator 0,47 (IPCC, 2006). As vantagens da adoção desses fatores estão na facilidade no cálculo do estoque de carbono e na ausência de custo. No entanto, a adoção de valores genéricos pode levar a superestimativas ou subestimativas de até $10 \%$ do estoque de carbono, visto que o teor de carbono varia entre $44,4 \%$ e $55,7 \%$, dependendo da espécie e do tecido avaliado (ZHANG et al., 2009; DALLAGNOL et al., 2011).

Estimativas errôneas de estoque de carbono podem ser evitadas se forem empregados teores de carbono determinados em laboratório. Esse método possibilita determinações precisas da concentração de carbono na biomassa, porém as análises envolvem um custo financeiro, o que muitas vezes impossibilita a realização das mesmas. O conhecimento do teor e estoque de carbono por espécie é de grande relevância, uma vez que projetos voltados à restauração e a fixação de carbono empregando vegetação nativa podem ser baseados em espécies ou grupos funcionais que apresentem maior capacidade de estocar carbono (WATZLAWICK et al., 2011).

Outra forma de obtenção do estoque de carbono é por meio de equações alométricas, que no caso de áreas em restauração tem grande importância, visto que em muitos casos o abate das árvores não é possível por questões técnicas e legais. Em geral, as equações alométricas apresentam como variáveis independentes características diretamente mensuráveis das árvores-amostra, como diâmetro e altura; já as variáveis dependentes são representadas, por exemplo, pelo volume, biomassa ou pela quantidade de carbono (CHAVE et al., 2005; SOARES et al., 2011). No entanto, com a utilização desse método há a possibilidade de erros significativos na estimativa de biomassa ou estoque de carbono se a equação usada não for apropriada para a área de estudo ou no caso da existência de árvores ocas ou de grande tamanho (CHAVE et al., 2004). Além disso, para áreas em restauração são escassos os estudos de modelagem da biomassa e estoque de carbono florestal não havendo, assim, um rol de equações alométricas publicadas na literatura (MIRANDA et al., 2011).

Todavia, nota-se que há uma grande carência de estudos em áreas de restauração, principalmente quando se trata da quantificação dos estoques de biomassa e carbono. Os poucos estudos disponíveis na literatura foram desenvolvidos no estado de São Paulo em áreas de domínio do Cerrado e Mata Atlântica com o uso de métodos indiretos (MELO; DURIGAN, 2006; MELO et al., 2009) e diretos (MIRANDA et al., 2011). Apenas dois desses estudos avaliaram áreas em restauração com mais de 20 anos (MELO; DURIGAN, 2006; MIRANDA et al., 2011).

Desse modo, trabalhos que visem avaliar a fixação de carbono em áreas em processo de restauração maduras são de grande relevância, principalmente pela escassez de estudos nesses locais. Áreas com maior tempo de restauração podem fornecer informações concisas sobre o acúmulo gradual de carbono por espécie e, assim, o potencial da área para remover o $\mathrm{CO}_{2}$ atmosférico. Vale ressaltar também, a necessidade de estudos em áreas em processo de restauração em diferentes situações, buscando a padronização nas metodologias de quantificação dos estoques de biomassa e carbono na vegetação a fim de que sejam geradas estimativas confiáveis e passíveis de comparação.

Diante do exposto, o presente trabalho foi realizado com o objetivo de estimar e comparar o estoque de carbono em uma área em restauração com 21 anos de idade no sul de Minas Gerais pela aplicação dos seguintes métodos indiretos: (M1) multiplicação da biomassa seca (BS) pelo teor de 
carbono obtido em laboratório, (M2) multiplicação da BS pelo fator 0,5 e (M3) uso de uma equação alométrica.

\section{MATERIAL E MÉTODOS}

\section{Descrição da área de estudo}

O presente estudo foi realizado em uma área em processo de restauração, de propriedade da Companhia Energética de Minas Gerais - CEMIG, localizada à jusante do reservatório de Camargos, na margem direita do Rio Grande, no município de Itutinga, MG (Figura 1). A vegetação natural da região onde a área de estudo está inserida é constituída de formações florestais, com predominância de Floresta Estacional Semidecidual (AZEVEDO, 1962). O clima da região é de transição entre Cwa e Cwb, de acordo com a classificação climática de Köppen (ANTUNES, 1986). A altitude local média é de $900 \mathrm{~m}$; a temperatura média anual, 19,4 ${ }^{\circ} \mathrm{C}$ e a precipitação média anual, 1.529,7 mm, com um período seco de abril a setembro. O tipo de solo predominante é o Latossolo Vermelho típico.

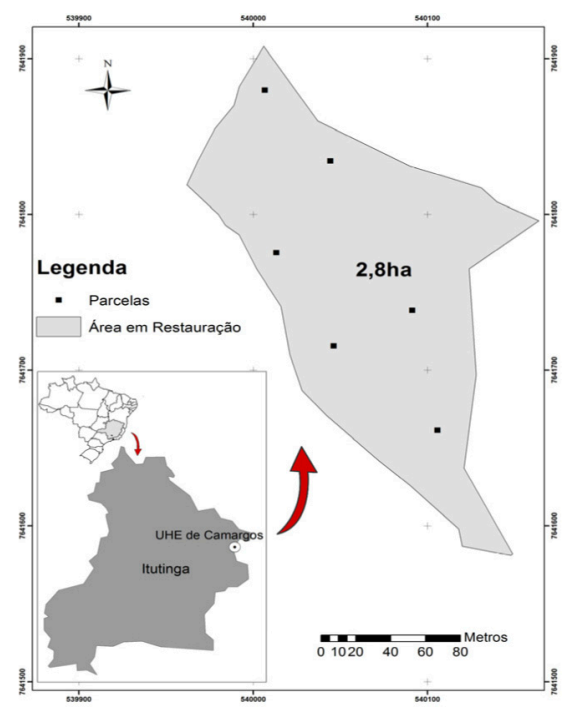

Figura 1. Mapa representativo da área em restauração, no entorno do reservatório de Camargos, Itutinga, MG. Figure 1. Representative map of the restored area around the reservoir of Camargos, Itutinga, MG.

A área em processo de restauração tem 21 anos, está situada nas coordenadas geográficas $21^{\circ} 19^{\prime} 28^{\prime \prime}$ de latitude Sul e $44^{\circ} 36^{\prime} 48^{\prime \prime}$ de longitude Oeste, e tem extensão de 2,8 hectares. Este local é uma área de empréstimo de terra que foi destinada à exploração na operação da construção da barragem da UHE Camargos, em meados da década de 50. Na área foi feita a supressão da vegetação e a remoção de cerca de cinco metros de perfil de solo. Com isso, o solo remanescente apresentou-se compactado, com baixa infiltração, formando bacias suscetíveis à erosão, além de possuir uma fertilidade muito baixa. No ano de 1992 iniciou-se a restauração florestal neste local, com o plantio de espécies florestais em quincôncio, no espaçamento de $1,5 \mathrm{~m} \mathrm{x} 3,0 \mathrm{~m}$, com base nos princípios de sucessão secundária (DAVIDE et al., 1993; FARIA, 2012). A relação das espécies florestais utilizadas é descrita por Faria (2012).

\section{Determinação da biomassa, teor de carbono e densidade básica da madeira}

Inicialmente foi executado um inventário florestal em julho de 2013 em seis parcelas permanentes de $20 \mathrm{~m}$ x $20 \mathrm{~m}$ estabelecidas no ano de 2010. Todos os indivíduos arbóreos com circunferência à altura do peito (CAP) $\geq 15 \mathrm{~cm}$ foram registrados, identificados botanicamente e mensurados quanto a altura total e CAP em 2010 e, posteriormente, em 2013. Para os indivíduos que apresentaram o fuste bifurcado, todos os fustes foram medidos e o diâmetro equivalente calculado (SOARES et al., 2011).

Posteriormente, calculou-se a estimativa da biomassa lenhosa seca do estrato arbóreo (BS) com base no diâmetro a altura do peito (DAP) e na altura total. Uma equação alométrica desenvolvida por Scolforo et al. (2008a) para uma Floresta Estacional Semidecidual nas bacias hidrográficas dos rios Grande e Piracicaba foi utilizada para esse fim (Eq. 1): 
Silva et al. - Estimativa do estoque de carbono por métodos indiretos em área de restauração florestal em Minas Gerais

$$
\begin{gathered}
\ln (\text { MS })=-10,9532786932+2,5464820134 \times \ln (D A P)+0,4667754371 \times \ln (H) \\
\left(\bar{R}^{2}=95,71 ; S_{y x}=41,74 \%\right)
\end{gathered}
$$

Em que:

$\mathrm{DAP}=$ diâmetro a $1,30 \mathrm{~m}$ do solo $(\mathrm{cm})$;

$\mathrm{H}=$ altura total $(\mathrm{m})$.

A seleção das espécies a serem amostradas para a determinação do teor de carbono e da densidade da madeira fundamentou-se na estrutura horizontal (Índice de Valor de Importância - IVI e área basal), sendo selecionadas 20 espécies (cerca de $40 \%$ do total) de alta importância ecológica e que contribuem com $87 \%$ da área basal total do local. O número mínimo de árvores amostradas por espécie foi proporcional ao valor da densidade relativa obtida com base no inventário florestal (SCOLFORO; THIERSCH, 2004). A seleção de quais indivíduos seriam amostrados foi realizada considerando-se a distribuição diamétrica das árvores registradas no inventário florestal, totalizando 50 árvores amostradas (15\% do total).

Nas árvores selecionadas foram retiradas amostras de lenho sem casca (baguetas) de $5 \mathrm{~mm}$ de diâmetro a 1,30 m do solo com o auxílio de um trado de incremento introduzido no tronco da árvore em pé até uma profundidade igual à metade do diâmetro. As amostras retiradas foram acondicionadas em sacos plásticos contendo água, os quais foram armazenados em caixa de isopor, para evitar a perda de umidade das amostras e para mantê-las inteiras. Cada saco foi previamente identificado com o número da amostra, o local e a data de coleta.

A densidade básica da madeira foi determinada em laboratório para as espécies tradadas, por meio da razão entre a biomassa (g) e o seu volume saturado $\left(\mathrm{cm}^{3} ;\right.$ Eq.: 2). A densidade básica da madeira de cada espécie amostrada foi obtida pela média aritmética das densidades das amostras da mesma espécie.

$$
D B M=\frac{\text { Biomassa sec } a(B s)}{\text { Volume saturado }(V s)} \quad[2]
$$

Em que:

DBM = Densidade básica da madeira, $\mathrm{g} \mathrm{cm}^{-3}$;

Bs = Biomassa ( seca em estufa), g;

$\mathrm{Vs}=$ Volume saturado, $\mathrm{cm}^{3}$.

Após a determinação da densidade, as amostras de madeira foram secas em estufa com circulação forçada de ar à temperatura de $103 \pm 2{ }^{\circ} \mathrm{C}$, até a estabilização do seu peso seco. A seguir, o material foi moído, macerado com o auxílio de um pistilo, peneirado em peneira de 60 mesh de granulometria e o pó retido nessa foi devidamente armazenado e enviado para análise do teor de carbono.

Um analisador do tipo TOC, modelo Vario TOC Cube, da marca Elementar foi utilizado nas análises de teor de carbono com sub amostras ( 3 a $5 \mathrm{mg}$ ) acondicionadas em cápsulas de estanho, as quais foram injetadas em forno a $950{ }^{\circ} \mathrm{C}$, para combustão a seco. O gás $\mathrm{CO}_{2}$ emitido por cada amostra foi quantificado por um detector de infravermelho do tipo NDIR e o carbono gerado foi relacionado com a massa (mg) da amostra avaliada.

O teor de carbono de cada espécie amostrada foi obtido pela média aritmética dos teores de carbono das amostras da mesma espécie. Já para as espécies as quais não se obteve essa informação, foi utilizado um valor médio obtido a partir das espécies amostradas.

\section{Estimativa do estoque de carbono pelo uso de métodos indiretos}

O estoque de carbono total foi estimado de três formas distintas. O primeiro método (M1), considerado no presente estudo como gerador da estimativa mais próxima do real, consistiu na multiplicação da biomassa estimada (BS; Eq. 1) pelo respectivo teor de carbono das amostras obtido em laboratório. No segundo método (M2), o estoque de carbono foi obtido por meio da multiplicação da BS estimada pelo fator 0,5. 
Alternativamente, como terceiro método (M3), estimou-se o estoque de carbono no estrato arbóreo (C) utilizando uma equação desenvolvida por Scolforo et al. (2008b) para Floresta Estacional Semidecidual das bacias hidrográficas dos rios Grande e Piracicaba (Eq. 3). Os valores de estoque de carbono obtidos foram extrapolados para hectare.

$$
\begin{gathered}
\ln (C)=-12,3034390630+2,6584231780 \times \ln (D A P)+0,5711719721 \times \ln (H) \\
\left(\bar{R}^{2}=97,25 ; S_{y x}=36,40 \%\right)
\end{gathered}
$$

Em que:

DAP = diâmetro a 1,30 $\mathrm{m}$ do solo $(\mathrm{cm})$;

$\mathrm{H}=$ altura total $(\mathrm{m})$.

O incremento periódico anual (IPA) do estoque de carbono na área de estudo também foi calculado para os períodos de 2010 e 2013 (Eq. 4).

$$
I P A_{c}=\frac{C_{2013}-C_{2010}}{n}
$$

Em que:

$\mathrm{IPA}_{\mathrm{c}}=$ Incremento periódico anual $\left(\mathrm{t} \mathrm{ha}{ }^{-1}\right)$;

$\mathrm{C}_{2013}=$ Estoque de carbono $\left(\mathrm{t} \mathrm{ha} \mathrm{a}^{-1}\right)$ em 2013;

$\mathrm{C}_{2010}=$ Estoque de carbono $\left(\mathrm{t} \mathrm{ha}^{-1}\right)$ em 2010;

$\mathrm{n}=$ intervalo de medição, no caso, 3 anos.

Para calcular o IPA os estoques de carbono nos anos de 2010 e 2013 foram obtidos conforme a equação 3 (SCOLFORO et al., 2008b), uma vez que a estimativa do estoque de carbono obtida no ano de 2010 foi realizada usando-se essa equação (FARIA, 2012).

\section{Análises estatísticas}

A análise de variância e o teste de Tukey ao nível de 5\% de significância foram executados para a comparação do estoque de carbono obtido a partir dos três métodos indiretos (M1, M2 e M3). Para tanto, inicialmente verificou-se a normalidade e a homogeneidade dos dados pelos testes de Shapiro-Wilk e Bartlett, respectivamente. Observou-se a partir dos resultados dos dois testes que os pressupostos básicos da estatística estão de acordo para a aplicação do teste de médias (Tukey). Pelo teste de Shapiro-Wilk, pode-se inferir que os resíduos de cada tratamento são provenientes de uma distribuição normal $(p=0,7980)$. O teste de homogeneidade de variâncias de Bartlett apontou que as variâncias dos métodos são homogêneas $(p=0,9258)$.

\section{RESULTADOS E DISCUSSÃO}

\section{Caracterização do estrato arbóreo}

No inventário florestal na área em restauração foram encontradas 48 espécies distribuídas em 26 famílias botânicas e uma densidade média de 1.283 indivíduos por hectare. Os valores médios do

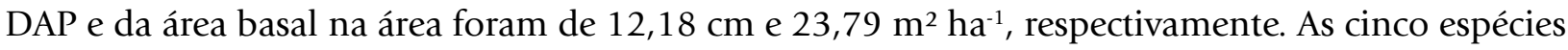
de maior ocorrência na área foram: Syzygium jambos (L.) Alston, Schinus terebinthifolius Raddi, Lithraea molleoides (Vell.) Engl, Peltophorum dubium (Spreng.) Taub. e Acacia mangium Willd (Tabela 1).

A Acacia mangium (Leguminosae Mimosoideae) foi a espécie de maior importância ecológica. Essa espécie apresenta rápido crescimento e, consequentemente, uma rápida cobertura do solo. Por isso, a A. mangium é comumente utilizada na restauração de áreas degradadas (BALIEIRO et al., 2004). No entanto, a espécie apresenta um ciclo de vida curto (FERREIRA et al., 2009), o que não é interessante, no longo prazo, se a restauração tiver como um de seus objetivos promover a fixação do carbono na biomassa. 
Silva et al. - Estimativa do estoque de carbono por métodos indiretos em área de restauração florestal em Minas Gerais

Tabela 1. Parâmetros fitossociológicos das espécies de maior Índice de Valor de Importância em área de restauração, no entorno do reservatório de Camargos, Itutinga, MG.

Table 1. Phytosociological parameters of the species with largest Importance Value Index in the restored area around the reservoir of Camargos, Itutinga, MG.

\begin{tabular}{|c|c|c|c|c|c|}
\hline Espécie & DR & DoR & FR & IVI & AB \\
\hline Acacia mangium Willd. & 5,84 & 22,04 & 0,03 & 27,91 & $\overline{1,26}$ \\
\hline Piptadenia gonoacantha (Mart.) J.F.M. & 2,27 & 15,68 & 0,03 & 17,99 & 0,90 \\
\hline Syzygium jambos (L.) Alston & 8,44 & 7,75 & 0,04 & 16,24 & 0,44 \\
\hline Anadenanthera peregrina (L.) Speg. & 4,87 & 8,17 & 0,04 & 13,09 & 0,47 \\
\hline Peltophorum dubium (Spreng.) Taub. & 6,17 & 6,16 & 0,05 & 12,38 & 0,35 \\
\hline Lithraea molleoides (Vell.) Engl. & 6,49 & 5,43 & 0,05 & 11,97 & 0,31 \\
\hline Schinus terebinthifolius Raddi & 7,47 & 3,51 & 0,05 & 11,03 & 0,20 \\
\hline Myrsine umbellata Mart. & 5,52 & 2,28 & 0,03 & 7,84 & 0,13 \\
\hline Enterolobium contortisiliquum (Vell.) M. & 3,25 & 3,60 & 0,03 & 6,87 & 0,21 \\
\hline Tapirira guianensis Aubl. & 4,55 & 1,33 & 0,04 & 5,92 & 0,08 \\
\hline Lafoensia vandelliana Cham. \& Schltdl. & 4,22 & 1,40 & 0,02 & 5,63 & 0,08 \\
\hline Cedrela fissilis Vell. & 3,90 & 1,63 & 0,03 & 5,56 & 0,09 \\
\hline Rapanea ferruginea (Ruiz \& Pav.) Mez. & 4,22 & 0,64 & 0,04 & 4,90 & 0,04 \\
\hline Nectandra nitidula Nees & 2,60 & 1,19 & 0,04 & 3,83 & 0,07 \\
\hline Ficus pertusa L.f. & 1,62 & 2,18 & 0,03 & 3,83 & 0,12 \\
\hline Cecropia pachystachya Trécul & 2,60 & 0,95 & 0,02 & 3,56 & 0,05 \\
\hline Copaifera langsdorffii Desf. & 2,27 & 0,84 & 0,03 & 3,15 & 0,05 \\
\hline Inga marginata Willd. & 0,97 & 1,70 & 0,01 & 2,68 & 0,10 \\
\hline Myrcia venulosa DC. & 1,95 & 0,45 & 0,03 & 2,42 & 0,03 \\
\hline Styrax leprosus Hook. \& Arn. & 1,95 & 0,29 & 0,03 & 2,26 & 0,02 \\
\hline
\end{tabular}

Nota: $D R$ = densidade relativa (\%); DoR = dominância relativa (\%); FR = frequência relativa (\%); IVI = índice de valor de importância (\%); $A B$ $=$ área basal $\left(\mathrm{m}^{2}\right)$.

\section{Biomassa, teor de carbono e densidade básica da madeira}

$\mathrm{Na}$ área em restauração a biomassa total foi de 109,40 tha $\mathrm{ta}^{-1}$. Os valores médios do teor de carbono e densidade básica da madeira para as espécies de maior importância ecológica estão apresentados na Tabela 2. As espécies que apresentaram o menor e o maior teor de carbono foram, respectivamente, Lafoensia vandelliana Cham. \& Schltdl. (43,7\%) e Anadenanthera peregrina (L.) Speg. (46,6\%). Já para a densidade básica da madeira as espécies que apresentaram o menor e o maior valor foram, respectivamente, Cecropia pachystachya Trécul $\left(0,31 \mathrm{~g} \mathrm{~cm}^{-3}\right)$ e Anadenanthera peregrina (L.) Speg. $\left(0,72 \mathrm{~g} \mathrm{~cm}^{-3}\right)$.

Em trabalho realizado por Amaro et al. (2013) em um fragmento de Floresta Estacional Semidecidual foram apresentados os teores de carbono para quatro espécies em comum com o presente trabalho: Anadenanthera peregrina (49,18\%), Copaifera langsdorffi $(47,25 \%)$, Piptadenia gonoacantha $(48,08 \%)$ e Tapirira guianensis $(46,54 \%)$. Observam-se que os valores de teor de carbono apresentados pelos autores são superiores aos encontrados nesse trabalho (Tabela 2). O teor de carbono na biomassa é resultado de uma interação entre os genes e o meio. Esse fato justifica as diferenças observadas entre espécies diferentes (LAMLOM; SAVIDGE, 2006). Para uma mesma espécie, além da interação com o meio, outras variáveis também devem ser consideradas para explicar as distintas concentrações de carbono na biomassa, tais como material genético, tipo de manejo e método de quantificação do teor de carbono e/ou da interação desses fatores (SETTE JR. et al., 2006).

É importante frisar também que os valores de teor de carbono para todas as espécies amostradas foram inferiores ao fator 0,5 (Tabela 2), o que destaca a importância de determinar com precisão essa variável para espécies ocorrentes em regiões e biomas distintos, pois diferenças aparentemente pouco expressivas podem repercutir em erros na quantificação do estoque de carbono.

Com relação à densidade básica da madeira, verificou-se que as espécies aqui estudadas apresentaram valores distintos aos disponíveis na Global Wood Density Database (ZANNE et al., 2009). Entretanto, comparações são prejudicadas em razão da variação natural da densidade devido a fatores abióticos e entre gêneros, entre espécies de um mesmo gênero e entre árvores de uma mesma espécie (LATORRACA; ALBUQUERQUE, 2000). Essa variação natural torna a densidade básica da madeira uma variável importante a ser incluída na modelagem da biomassa e carbono florestal para melhorar a confiabilidade das estimativas de biomassa e carbono geradas pelo uso de equações alométricas (CHAVE et al., 2004; CHAVE et al., 2005). Desse modo, a determinação da densidade 
básica da madeira e do teor de carbono deve ser feita em laboratório sempre que possível para minimizar erros e possibilitar o ajuste futuro de equações para estimar o estoque de carbono na vegetação a partir de variáveis dendrométricas (PANDEY et al., 2014).

Tabela 2. Valores médios do teor de carbono e densidade básica da madeira das espécies amostradas na área em restauração, no entorno do reservatório de Camargos, Itutinga, MG.

Table 2. Mean values of carbon content and basic wood density of species sampled in the restored area around the reservoir of Camargos, Itutinga, MG.

\begin{tabular}{|c|c|c|}
\hline Espécie & Teor de Carbono (\%) & Densidade Básica da Madeira $\left(\mathrm{g} \mathrm{cm}^{-3}\right)$ \\
\hline Acacia mangium Willd. & 46,30 & 0,56 \\
\hline Anadenanthera peregrina (L.) Speg. & 46,60 & 0,72 \\
\hline Cecropia pachystachya Trécul & 43,80 & 0,31 \\
\hline Cedrela fissilis Vell. & 44,20 & 0,41 \\
\hline Copaifera langsdorffii Desf. & 45,40 & 0,66 \\
\hline Enterolobium contortisiliquum (Vell.) Morong & 44,00 & 0,45 \\
\hline Ficus pertusa L.f. & 43,90 & 0,41 \\
\hline Inga marginata Willd. & 46,40 & 0,65 \\
\hline Lafoensia vandelliana Cham. \& Schltdl. & 43,70 & 0,58 \\
\hline Lithraea molleoides (Vell.) Engl. & 44,20 & 0,61 \\
\hline Myrcia venulosa DC. & 44,80 & 0,66 \\
\hline Myrsine umbellata Mart. & 44,20 & 0,62 \\
\hline Nectandra nitidula Nees & 45,10 & 0,41 \\
\hline Peltophorum dubium (Spreng.) Taub. & 44,70 & 0,55 \\
\hline Piptadenia gonoacantha (Mart.) J.F.Macbr. & 45,20 & 0,57 \\
\hline Rapanea ferruginea (Ruiz \& Pav.) Mez. & 44,40 & 0,58 \\
\hline Schinus terebinthifolius Raddi & 44,80 & 0,52 \\
\hline Styrax leprosus Hook. \& Arn. & 45,40 & 0,59 \\
\hline Syzygium jambos (L.) Alston & 45,80 & 0,67 \\
\hline Tapirira guianensis Aubl. & 45,10 & 0,46 \\
\hline Média (DP) & $44,90(0,88)$ & $0,55(0,11)$ \\
\hline Intervalo de confiança & $44,90 \pm 0,41$ & $0,55 \pm 0,05$ \\
\hline
\end{tabular}

Nota: DP = desvio padrão.

\section{Comparação dos métodos indiretos para estimativa do estoque de carbono}

$\mathrm{Na}$ área em restauração o estoque de carbono total foi de 49,73 t ha-1. Na Tabela 3 estão apresentados os valores médios de estoque de carbono obtidos pelos três métodos de estimativa (M1, M2 e M3) testados no presente estudo. Verificou-se que o método M2 e o M3 foram estatisticamente iguais, apresentando as maiores estimativas de estoque de carbono. Por outro lado, o método M1 foi estatisticamente diferente dos demais métodos e apresentou a menor estimativa do estoque de carbono. Dessa forma, observa-se que o uso da equação alométrica (M3) e de um teor de carbono genérico (M2) levou a superestimativa do estoque de carbono na área avaliada.

Tabela 3. Valores médios de estoque de carbono $\left(\mathrm{t} \mathrm{ha} \mathrm{a}^{-1}\right)$, obtidos pelos diferentes métodos indiretos.

Table 3. Mean values of carbon stock $\left(\mathrm{t} \mathrm{ha}^{-1}\right)$ obtained by different indirect methods.

\begin{tabular}{lc}
\hline Métodos & Estoque de carbono (t ha-1) \\
\hline BS $\times 0,5$ & 55,65 a \\
Scolforo et al. (2008b) & $54,70 \mathrm{a}$ \\
BS x Teor de carbono & $49,73 \mathrm{~b}$ \\
\hline CV (\%) & $\mathbf{4 , 0}$ \\
\hline
\end{tabular}

*Médias seguidas de letras iguais na coluna não diferem entre si pelo teste de Tukey ao nível de $5 \%$ de significância.

Koehler et al. (2005); Corte et al. (2012) afirmam que a utilização de equações e de teores de carbono não apropriados levam a estimativas consideravelmente diferentes dos valores obtidos em campo. Dessa forma, o uso do teor de carbono obtido em laboratório é recomendável e contribui para aumentar a precisão das estimativas de estoque de carbono geradas a partir de métodos indiretos de obtenção da biomassa. Em áreas em processo de restauração, nas quais muitas vezes não é possível realizar o abate das árvores, esse fato é de grande relevância, visto que se insere na estimativa uma maior especificidade ao associar um valor de teor de carbono que reflete as características daquele ambiente. 
O uso de uma equação alométrica para estimar o estoque de carbono, mesmo tendo sido desenvolvida para uma mesma tipologia florestal, pode gerar valores não representativos sobre a capacidade das árvores em estocar carbono, uma vez que, principalmente condições ambientais como clima e solo podem influenciar no estoque de biomassa e carbono. Assim, uma alternativa é incluir no modelo de predição variáveis dendrométricas que reflitam as condições do ambiente, tais como a densidade básica da madeira (CHAVE et al., 2005; WILLIAMSON; WIEMANN, 2010).

Torres et al. (2013), em um estudo que envolveu o uso de equações regionais e de uma metodologia do IPCC para quantificar a biomassa e o armazenamento de carbono em uma Floresta Estacional Semidecidual, verificaram que metodologia do IPCC apresentou tendência de subestimar a biomassa e o carbono estocado, em comparação com as equações regionais. Os autores destacaram também a necessidade da realização de estudos em outros ecossistemas florestais e da padronização das metodologias de quantificação dos estoques de biomassa e carbono na vegetação, para aumentar a qualidade dos bancos de dados e possibilitar a comparação entre diferentes estudos.

\section{Incremento do estoque de carbono entre os períodos de 2010 e 2013}

O incremento periódico anual médio do estoque de carbono, nos anos de 2010 a 2013 foi de $3,72 \mathrm{t} \mathrm{ha}^{-1}$ ano $^{-1}$ na área em restauração. Já o incremento médio anual (IMA) do estoque de carbono foi de 2,60 $\mathrm{tha}^{-1} \mathrm{ano}^{-1}$.

Melo e Durigan (2006) ao avaliarem a fixação de carbono em reflorestamentos de matas ciliares implantados em região de Cerrado, aos nove anos, constataram que esses apresentaram um incremento médio anual de fixação de carbono de 1,5 t ha-1 ano-1. Melo et al. (2009) ao quantificarem os estoques de biomassa e carbono em áreas de Cerrado em restauração, aos seis anos, verificaram um IMA de 2,5 t ha-1 ano $^{-1}$. Tal fato evidencia que a área, apesar de já estar há 21 anos em processo de restauração possui um alto incremento em estoque de carbono e, assim, vem conseguindo cumprir as suas funções ambientais como sumidouro de carbono.

O incremento em biomassa e carbono em florestas tropicais em sucessão é influenciado pela idade e grupo sucessional das espécies presentes (SHIMAMOTO et al., 2014). Kenzo et al. (2010) ao estudarem a biomassa e o carbono em florestas secundárias na Malásia verificaram uma alta taxa de acúmulo de carbono nos primeiros dez anos de sucessão, com a redução significativa dessa taxa após a primeira década. Já em áreas em restauração em Porto Rico, Marin-Spiotta et al. (2007) registraram altas taxas de acúmulo de biomassa e carbono durante os primeiros 20 anos. Tal fato também foi constatado no presente estudo.

Além da idade, a composição de espécies usada na restauração também influencia no incremento em carbono. Em geral, espécies de crescimento lento e com ciclos de vida longos tendem a acumular mais carboidratos e, assim, carbono na biomassa do que espécies de ciclo de vida curto. Entretanto, em termos anuais, espécies de crescimento rápido irão acumular carbono a uma taxa maior do que espécies de crescimento lento (SHIMAMOTO et al., 2014). Dessa forma, o balanço entre espécies pertencentes a diferentes grupos ecológicos é o mais recomendado para manter a estrutura do ecossistema e o sequestro e estoque de carbono na biomassa florestal.

Outros fatores também devem ser considerados na avaliação do potencial de fixação de carbono em áreas florestais tropicais, tais como o histórico de perturbação e condições climáticas e edáficas (NGO et al., 2013). Desse modo, faz-se necessário pesquisas e estabelecimento de parâmetros e informações para cada localidade baseadas nas características da comunidade florestal estabelecida.

\section{CONCLUSÕES}

Na comparação dos teores médios de carbono obtidos em laboratório (M1) com o fator 0,5 (M2), constatou-se que esse superestima em $11 \%$ o teor de carbono das espécies avaliadas. Com relação aos métodos de estimativa do estoque de carbono analisados no presente estudo, verificou-se que o uso da equação alométrica e de um teor de carbono genérico levaram a superestimativas, respectivamente, de $10 \%$ e $12 \%$ do estoque de carbono na área. Dessa forma, o uso do teor de carbono determinado em laboratório contribuiu para aumentar a precisão da estimativa de estoque de carbono. 


\section{REFERÊNCIAS BIBLIOGRÁFICAS}

ALMEIDA, E. M.; CAMPELO JR., J. H.; FINGER, Z. Determinação do estoque de carbono em Teca (Tectona grandis LF) em diferentes idades. Ciência Florestal, Santa Maria, v. 20, n. 4, p. 559-568, 2010.

AMARO, M. A.; SOARES, C. P. B.; SOUZA, A. L.; LEITE, H. G.; SILVA, G. F. Estoque volumétrico, de biomassa e de carbono em uma Floresta Estacional Semidecidual em Viçosa, Minas Gerais. Revista Árvore, Viçosa, v. 37, n. 5, p. 849-857, 2013.

ANTUNES, F. Z. Caracterização climática do Estado de Minas Gerais. Informe Agropecuário, Belo Horizonte, v. 12, n. 138 , p. 9-13, 1986.

ASHTON, M. S.; TYRRELL, M. L.; SPALDING, D.; GENTRY, B. Managing forest carbon in a changing climate. Londres: Springer, 2012. 425 p.

AZEVEDO, L. G. Tipos de vegetação do Sul de Minas e Campos da Mantiqueira (Brasil). Anais da Academia Brasileira de Ciências, Rio de Janeiro, v. 34, n. 2, p. 225-234, 1962.

BALIEIRO, F. C.; DIAS, L. E.; FRANCO, A. A.; CAMPELLO, E. F. C.; FARIA, S. M. Acúmulo de nutrientes na parte aérea, na serapilheira acumulada sobre o solo e decomposição de filódios de Acacia mangium Willd. Ciência Florestal, Santa Maria, v. 14, n. 1, p. 59-65, 2004.

CHAVE, J.; ANDALO, C.; BROWN, S.; CAIRNS, M. A.; CHAMBERS, J. Q.; EAMUS, D.; FÖLSTER, H.; FROMARD, F.; HIGUCHI, N.; KIRA, T.; LESCURE, J. P.; NELSON, B.W.; OGAWA, H.; PUIG, H.; RIÉRA, B., YAMAKURA, T. Tree allometry and improved estimation of carbon stocks and balance in tropical forests. Oecologia, Berlin, v. 145, n. 1, p. 87-99, 2005.

CHAVE, J.; CONDIT, R.; AGUILAR, S.; HERNANDEZ, A.; LAO, S.; PEREZ, R. Error propagation and scaling for tropical forest biomass estimates. Philosophical Transactions of the Royal Society B, Londres, v. 359, n. 1443, p. 409-420, 2004.

CORTE, A. P. D.; SILVA, F; SANQUETTA, C. R. Fator de expansão de biomassa e razão de raízes-parte aérea para Pinus spp. plantadas no Sul do Brasil. Floresta, Curitiba, v. 42, n. 4, p. 755-768, 2012.

DALLAGNOL, F. S.; MOGNON, F.; SANQUETTA, C. R.; CORTE, A. P. D. Teores de carbono de cinco espécies florestais e seus compartimentos. Floresta e Ambiente, Seropédica, v. 18, n. 4, p. 310-316, 2011.

DAVIDE, A. C.; SCOLFORO, J. R. S.; FARIA, J. M. R. Adaptação de 12 espécies florestais em área de empréstimo. In: CONGRESSO FLORESTAL PANAMERICANO, 1; CONGRESSO FLORESTAL BRASILEIRO, 7. 1993, Curitiba. Anais... Curitiba: SBS/SBEF, 1993. p. 754.

FARIA, R. A. V. B. Estoque de carbono e atributos florísticos e edáficos de ecossistemas florestais em processo de restauração. 2012. 168 p. Tese (Doutorado em Engenharia Florestal) - Universidade Federal de Lavras, Lavras, 2012.

FERREIRA, W. C.; BOTELHO, S. A.; DAVIDE, A. C.; FARIA, J. M. R. Estabelecimento de mata ciliar às margens do reservatório da Usina Hidrelétrica de Camargos, MG. Ciência Florestal, Santa Maria, v. 19, n. 1, p. 69-81, 2009.

HOUGHTON, R. Aboveground forest biomass and the global carbon balance. Global Change Biology, London, v. 11, n. 6, p. 945-958, 2005.

IPCC - INTERGOVERNMENTAL PANEL ON CLIMATE CHANGE. Good practice guidance for land use, landuse change and forestry. Japão: IGES, 2003.

Sci. For., Piracicaba, v. 43, n. 108, p. 943-953, dez. 2015 DOI: dx.doi.org/10.18671/scifor.v43n108.18 
Silva et al. - Estimativa do estoque de carbono por métodos indiretos em área de restauração florestal em Minas Gerais

IPCC - INTERGOVERNMENTAL PANEL ON CLIMATE CHANGE. Guidelines for National Greenhouse Gas Inventories, Prepared by the National Greenhouse Gas Inventories Programme. Japão: IGES, 2006.

KENZO, T.; ICHIEB, T.; HATTORI, D.; KENDAWANG, J. J.; SAKURAI, K.; NINOMIYAE, I. Changes in aboveand belowground biomass in early successional 47 tropical secondary forests after shifting cultivation in Sarawak, Malaysia. Forest Ecology and Management, Amsterdam, n. 260, n. 5, p. 875-882, 2010.

KOEHLER, H. S.; WATZLAWICK, L. F.; KIRCHNER, F. F.; VALÉRIO, A. F. Fontes de erros nas estimativas de biomassa e carbono fixado na Floresta Ombrófila Mista. Biomassa \& Energia, Viçosa, v. 2, n. 1, p. 69-77, 2005.

LAMLOM, S. H.; SAVIDGE, R. A. Carbon content variation in boles of mature sugar maple and giant sequoia. Tree physiology, Victoria, v. 26, n. 4, p. 459-468, 2006.

LATORRACA, J. V. F; ALBUQUERQUE, C. E. C. Efeito do rápido crescimento sobre as propriedades da madeira. Floresta e Ambiente, Seropédica, v. 7, n. 1, p. 279-291, 2000.

MARIN-SPIOTTA, E.; OSTERTAG, R.; SILVER, W. L. Long-term patterns in tropical reforestation: plant community composition and aboveground biomass accumulation. Ecology Applied, Germany, n. 17, n. 3, p. 828-839, 2007.

MELO, A. C. G.; DURIGAN, G. Fixação de carbono em reflorestamentos de matas ciliares no Vale do Paranapanema, SP, Brasil. Scientia Forestalis. Piracicaba, n. 71, p. 149-154, 2006.

MELO, A. C. G.; SOUSA, H.; CONTIERI, W. A.; MALICIA, L. C. Biomassa, fixação de carbono e estrutura da vegetação de cerrado em restauração aos seis anos, Assis, SP. Revista do Instituto Florestal, São Paulo, v. 21, n. 1, p. 73-78, 2009.

MIRANDA, D. L. C.; MELO, A. C. G.; SANQUETTA, C. R. Equações alométricas para estimativa de biomassa e carbono em árvores de reflorestamentos de restauração. Revista Árvore, Viçosa, v. 35, n. 3, p. 679-689, 2011.

NGO, K. M.; TURNER, B. L.; MULLER-LANDAU, H. C.; DAVIES, S. J.; LARJAVAARA, M.; HASSAN, N. F. B. N.; LUM, S. Carbon stocks in primary and secondary tropical forests in Singapore. Forest Ecology and Management, Amsterdam, n. 296 p. 81-89, 2013.

PAIVA, A. O.; REZENDE, A. V.; PEREIRA, R. S. Estoque de carbono em cerrado sensu stricto do Distrito Federal. Revista Árvore, Viçosa, v. 35, n. 3, p. 527-538, 2011.

PANDEY, R.; HOM, S. K.; HARRISON, S.; YADAV, V. K. Mitigation potential of important farm and forest trees: a potentiality for clean development mechanism afforestation reforestation (CDMA R) project and reducing emissions from deforestation and degradation, along with conservation and enhancement of carbon stocks (REDD+). Mitigation and Adaptation Strategies for Global Change, 2014. DOI 10.1007/ s11027-014-9591-2. Disponível em: < http://link.springer.com/article/10.1007\%2Fs11027-014-9591-2 <. Acesso em: 30 jun 2015.

RIBEIRO, S. C.; JACOVINE, L. A. G.; SOARES, C. P. B.; MARTINS, S. V.; SOUZA, A. L.; NARDELLI, A. M. B. Quantificação de biomassa e estimativa de estoque de carbono em uma floresta madura no município de Viçosa, Minas Gerais. Revista Árvore, Viçosa, v. 33, n. 5, p. 917-926, 2009.

SANQUETTA, C. R.; BALBINOT, R. Metodologias para determinação de biomassa florestal. In: SANQUETTA, C. R.; BALBINOT, R.; ZILIOTTO, M. A. B. (Eds.). Fixação de carbono: atualidades, projetos e pesquisas. Curitiba: UFPR/Ecoplan, 2004. p.77-93. 
SCOLFORO, J. R. S.; RUFINI, A. L.; MELlO, J. M.; TRUGILHO, P. F.; OlIVEIRA, A. D.; SILVA, C. P. C. Equações para o peso de matéria seca das fisionomias, em Minas Gerias. In: SCOLFORO, J. R.; OLIVEIRA, A. D.; ACERBI JR., F. W. (Eds.). Inventário Florestal de Minas Gerais - Equações de volume, peso de matéria seca e carbono para diferentes fisionomias da flora nativa. Lavras: UFLA, 2008a. Cap. 3, p. 103-114.

SCOLFORO, J. R. S.; RUFINI, A. L.; MELLO, J. M.; OLIVEIRA, A. D.; SILVA, C. P. C. Equações para a quantidade de carbono das fisionomias, em Minas Gerais. In: SCOLFORO, J. R.; OLIVEIRA, A. D.; ACERBI JR., F. W. (Eds.). Inventário Florestal de Minas Gerais - Equações de volume, peso de matéria seca e carbono para diferentes fisionomias da flora nativa. Lavras: UFLA, 2008b. Cap. 9, p. 198-216.

SCOLFORO, J. R. S.; THIERSCH, C. R. Biometria florestal: medição, volumetria e gravimetria. Lavras: UFLA/ FAEPE, 2004. 285 p.

SETTE JR., C. R.; NAKAJIMA, N. Y.; GEROMINI, M. P. Captura de carbono orgânico em povoamentos de Pinus taeda L. na região de Rio Negrinho, SC. Floresta, Curitiba, v. 36, n. 1, p. 33-44, 2006.

SHIMAMOTO, C. Y.; BOTOSSO, P. C.; MARQUES, M. C. M. How much carbon is sequestered during the restoration of tropical forests? Estimates from tree species in the Brazilian Atlantic forest. Forest Ecology and Management, Amsterdam, n. 329, p. 1-9, 2014.

SOARES, C. P. B.; OLIVEIRA, M. L. R. Equações para estimar a quantidade de carbono na parte aérea de árvores de eucalipto em Viçosa, Minas Gerais. Revista Árvore, Viçosa, v. 26, n. 5, p. 533-539, 2002.

SOARES, C. P. B.; PAULA NETO, F.; SOUZA, A. L. Dendrometria e Inventário Florestal. Viçosa: Ed. UFV, 2011. $278 \mathrm{p}$.

SOUZA, J. T.; FIORENTIN, L. D. Quantificação da biomassa e do carbono em povoamento de Eucalyptus grandis W. Hill ex Maiden, em Santa Maria, RS. Unoesc \& Ciência - ACET, Joaçaba, v. 4, n. 2, p. 253-262, 2013.

TORRES, C. M. M. E.; JACOVINE, L. A. G.; SOARES, C. P. B.; OLIVEIRA NETO, S. N.; SANTOS, R. D.; CASTRO NETO, F. Quantificação de biomassa e estocagem de carbono em uma Floresta Estacional Semidecidual, no Parque Tecnológico de Viçosa, MG. Revista Árvore, Viçosa, v. 37, n. 4, p. 647-655, 2013.

WATZLAWICK, L. F.; EBLING, A. A.; RODRIGUES, A. L.; VERES, Q. J. I.; LIMA, A. M. Variação nos teores de carbono orgânico em espécies arbóreas da Floresta Ombrófila Mista. Floresta e Ambiente, Seropédica, v. 18, n. 3, p. 248-258, 2011.

WILLIAMSON, G. B.; WIEMANN, M. C. Age-dependent radial increases in wood specific gravity of tropical pioneers in Costa Rica. Biotropica, Lawrence, v. 42, n. 5, p. 590-597, 2010.

ZANNE, A. E.; LOPEZ-GONZALEZ, G.; COOMES, D. A.; ILIC, J.; JANSEN, S.; LEWIS, S. L.; MILLER, R. B.; SWENSON, N. G.; WIEMANN, M. C.; CHAVE, J. Global wood density database. 2009. Disponível em: < http://hdl.handle.net/10255/dryad.235 >. Acesso em: 12 jan. 2014.

ZHANG, Q.; WANG, C.; WANG, X.; QUAN, X. Carbon concentration variability of 10 Chinese temperate species. Forest Ecology and Management, Amsterdam, v. 258, n. 5, p. 722-727, 2009.

Recebido em 05/06/2014

Aceito para publicação em 15/06/2015

Sci. For., Piracicaba, v. 43, n. 108, p. 943-953, dez. 2015 DOI: dx.doi.org/10.18671/scifor.v43n108.18 
